\title{
Über einige durch Digitaliskörper hervorgerufene Haltungsanomalien.
}

\author{
Von \\ Nobuyuki Takase. \\ (高 瀨 信 之) \\ (Aus dem Pharmakologischen Institut der Kaiserlichen Tohoku \\ Universität zu Sendai. Director: Prof. S.Yagi.)
}

Santess on und Strindberg ${ }^{1)}$ fanden bei Kaninchen nach intravenöser Darreichung einer tödlichen Dosis Gitalin, Digalen oder Digitalisinfus ausser Erregungserscheinungen der Medulla oblongata, wie beschleunigte Atmung, Zittern, Krampfbewegungen und Pulsverlangsamung, die schon von früheren Forschern bei Versuchen mit verschiedenen Digitaliskörpern bemerkt worden waren, noch eine Schwächung und Lähmung gewisser MuskeJgruppen, vor allem der Nackenmuskeln, die weder vorher noch nach her von anderen Autoren beschrieben worden sind. Über die Folgeerscheinungen der Nackenmuskellähmung brachten sie eine kurze Beschreibung, erwähnten aber leider nichts vom Einfluss der Lähmung der anderen Muskelgruppen auf die Körperhaltung. Auf Grund ihrer Beobachtung, nach der die Nackenmuskellähmung auch bei subduraler Injektion des Gitalins erzielt werden konnte, sahen sie die Ursache dieser Vergiftungserscheinung in der Wirkung auf das Zentralnervensystem, wobei sie jedoch die Frage offen liessen, welche Stelle des Nervensystems hierbei vom Gift angegriffen wird. Die vorliegende Untersuchung wurde zu dem $Z$ weck ausgeführt, die durch irgendeine herzwirksame Substanz aus den Digitalisblättern herbeigeführten Haltungsanomalien genauer zu beobachten und, wenn möglich, ihre Entstehungsweise zu analysieren. Gelegentlich wurde auch festgestellt, ob herzwirksame Substanzen anderer Drogen ebenso auf die Körperhaltung einwirken, wie die aus den Digitalisblättern gewonnenen.

Unsere eigenen Versuche wurden ebenso wie die der genannten

1) Santesson u. Strindberg, Wien. med. Wochenschr., 1916, 541. 
Forscher ausschliesslich an Kaninchen angestellt. Als zu prüfende Substanz wurde zuerst das Digitoxin gewählt, wovon eine $0,5 \%$ ige alkoholische Lösung hergestellt wurde. $0,1 \mathrm{ccm}$ dieserLösung $(0,05 \mathrm{mg})$ wurde mit Wasser auf $1 \mathrm{ccm}$ verdünnt und in die Vene eines Kaninchens von etwa $2 \mathrm{~kg}$ Körpergewicht injiziert. Die Injektion wurde in Intervallen von 15 Minuten solange wiederholt, bis sich das erste Anzeichen der Anomalien in der Körperhaltung einstellte. Die geringste Menge des Digitoxins, die hierzu nötig war, war je nach den einzelnen Versuchstieren ziemlich verschieden und schwankte zwischen 0,15 und $0,4 \mathrm{mg}$ pro Tier, lag aber in den meisten Fällen bei $0,3 \mathrm{mg}$.

Das erste Anzeichen der Haltungsanomalien setzte meistens beinahe gleichzeitig mit einer Atembeschleunigung und einer Pulsverlangsamung ein und bestand, wie der frühere Forscher bereits berichtete, in einem Hängenlassen des Kopfes, das dann allmählich immer auffallender wurde, bis der Kopf schliesslich mit der Schnauze den Boden berührte, wobei sich die Längsachse des Kopfes ungefähr senkrecht zum letzteren einstellte. Das Tier schien manchmal den herabhängenden Kopf wieder in seine frühere Stellung bringen zu wollen, was ihm anfänglich wenn auch ungenügend und nur für kurze Zeit, aber später nicht mehr gelang. Dann neigte sich der Kopf mehr und mehr nach hinten und sank $\mathrm{z}$ wischen die beiden etwas gestreckten Vorderbeine, sodass die Ohren sich nach vorn und die Schnauze nach der Bauchseite hin richteten. Der Hals leistete dabei gegen ein Aufheben des Kopfes an den Ohren keinen starken Widerstand und der Kopf sank bald nach dem Loslassen der Ohren herab und schlug mit der Schnauze hörbar auf den Boden auf. Hängte man das Tier an den vier Beinen mit dem Rücken nach unten auf, so hing der Kopf schlaff herunter, während die Beine einen normalen Widerstand zeigten. Wenn die Giftdarreichung in der Zeit unterbrochen wurde, in der das erste Anzeichen der Haltungsanomalien auftrat, so ging diese $\mathrm{Zu}-$ standsänderung in wenigen Fällen nach einigen Stunden zurück. Bestenfalls konnte sie aber über 24 Stunden fortbestehen, ohne dabei von Haltungsveränderungen der anderen Körperteile begleitet zu sein. Dabei sah man oft, dass das Tier mit herabhängendem Kopf umherhüpfte, als ob es nach Futter suchte. Gab man ihm Futter, indem man dieses auf dem Boden verstreute, so wurde es vom Tier, wenn auch nur mit Mühe, gefressen. Wenn das Futter in einen Kasten mit einer Höhe von etwa $5 \mathrm{~cm}$ hineingelegt wurde, so konnte das Tier es nicht mehr fressen, da es nicht imstande war, die Schnauze in den Kasten 
hineinzubringen. In den meisten Versuchen gesellte sich zu der eben erwähnten Haltungsanomalie noch eine solche der vorderen Extremitäten, die kurze oder lange Zeit nach dem Auftreten der ersteren zum Vorschein kam. Das Tier setzte dann die Vorderläufe auf dem Boden auf, sodass die vordere Körperhälfte mit der Brust den Boden berührte, wobei sich der Kopf auf oder zwischen die vorderen Extremitäten legte, während sich die hintere Körperhälfte noch aufrecht hielt. Dabei machte das Tier freiwillig keine grösseren Bewegungen und neigte dazu, in umgelegter Stellung zu verharren. Auf starke mechanische Reizung hin suchte es die vordere Körperhälfte wieder aufzurichten und zu entfliehen, was jedoch stets vergeblich war. Inzwischen begannen die hinteren Extremitäten, die Unterschenkel an den Kniegelenken und die Füsse an den Fussgelenken sich zu strecken, sodass die Füsse später ausserordentlich weit nach vorn geschoben wurden. Infolgedessen sank die hintere Körperhälfte immer tiefer, um sich dann schliesslich auf die Seite zu legen. Die Atembewegungen waren dabei unregelmässig und zuweilen dyspnoisch und die Herzschläge sehr langsam. Willkürliche Bewegungen waren fast gar nicht bemerkbar und die Reflexbewegungen konnten durch starke mechanische Reizung nur schwer ausgelöst werden. Die nebenstehende Figur lässt dieses Verhalten deutlich erkennen.

Versuche mit dem G-Strophanthin, dem Adnidin und dem Rhodein ergaben, dass all diese Glukoside ähnliche Anomalien der Körperhaltung hervorriefen, wie das Digitoxin. Nach wenigen Versuchsresultaten schienen die einzelnen Haltungsanomalien beim Adnidin in längeren Zeitintervallen als beim Digitoxin nacheinander aufzutreten, während sie
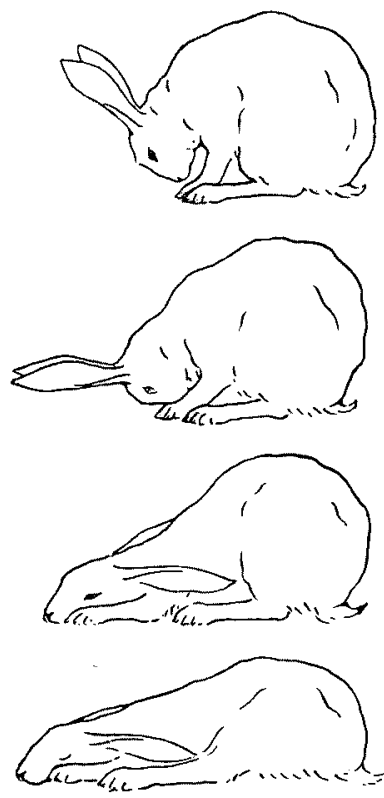

Fig. Reihenfolge der durch intravenöse Digitoxindarreichung herbeigeführten $\mathrm{Hal}$ tungsanomalien eines Kaninchens.

beim Strophanthin und Rhodein vielmehr gleichzeitig zum Vorschein zu kommen pflegten.

Nach diesen Versuchen ist es wahrscheinlich, dass die Digitaliskörper von verschiedener Herkunft auch die Eigenschaft haben, 
charakteristische Haltungsanornalien zu erzeugen. Nach $\mathrm{Narum} \mathrm{i}^{2>}$ scheinen die aus der $Z$ wiebel von Fritillaria verticillata, Wild. gewonnenen Alkaloide, das Verticin, das Verticillin und das Fritillarin, ebenfalls Haltungsanomalien des Kopfes und derExtremitäten hervorzurufen, die aber in umgekehrter Reihenfolge wie bei den Digitaliskörpern auftreten, sodass bei ihnen die diesen eigentümlichen Haltungsanomalien des Kopfes nicht bemerkt werden. Jedenfalls ist es sehr interessant, auf die Entstehung der oben geschilderten, durch die Digitaliskörper hervorgerufenen Haltungsanomalien näher einzugehen.

$\mathrm{Da}$ die eigentümliche Haltungsanomalien des Kopfes und der Extremitäten nach intravenöser Injektion vom Digitoxin auch bei Kaninchen auftraten, denen das Grosshirn mit dem Thalamus 24 Stunden vorher herausgeschnitten worden war, ist es sicher, dass weder das Grosshirn noch der Thalamus bei der Entstehung der genannten Haltungsanomalien eine wichtige Rolle spielen. Auch der Vierhügel scheint mit ihrem Zustandekommen in keinem wesentlichen Zusammenhang zu stehen, denn weder eine Bepinselung seiner Oberfläche mit einer $0,05 \%$ igen Digitoxinlösung noch eine Injektion mit dieser Lösung in die Oberflächenschicht verschiedener Stellen desselben verursachten solche Erscheinungen, während diese Behandlung nicht selten ein Rückwärtsdrehen des Kopfes veranlassten. Danach ist es wahrscheinlich, dass die Haltungsanomalien gerade wie beim Verticin von der Wirkung des Giftes auf das Kleinhirn abhängig sind. Um dies festzustellen, wurden die folgenden Versuche ausgeführt, bei denen die Giftlösung in das blossgelegte Kleinhirn des Kaninchens injiziert wurde.

Wurde die Injektion mit $0,01 \mathrm{ccm}$ einer $0,05 \%$ igen Lösung in die Kleinhirnsubstanz etwa $2 \mathrm{~mm}$ tief ausgeführt, so trat nach kurzer Zeit je nach den Injektionsstellen bald die eine, bald die andere Haltungsanomalie auf, die innerhalb einer Stunde vollständig zurückgingen. Von den geprüften Stellen schienen aber nur die folgenden an der Entstehung der in Frage kommenden Haltungsanomalien beteiligt zu sein. Bei der Injektion in die Mitte des Lobuli simp., das von Bolk ${ }^{3}$ ) und Rynberk ${ }^{4)}$ als der Sitz des Koordinationszentrums für die Nakkenmuskeln betrachtet worden ist, trat nach vorübergehenden Rückwärtsdrehbewegungen des Kopfes eine Verschiebung der vorderen Extremitäten nach vorn ein, sodass dann die Vorderläufe und die Brust

2) Narumi, Tohoka journ. of exp. Med., 1936, 28, 16.

3) Bolk, Das Cerebellum der Säugetiere, Berlin 1906, 306

4) R y n berk, Arch. di fisiolog., 1904, 1, 569. 
den Boden berührten, während sich der Kopf und die hinteren Extremitäten fast in normaler Haltung befanden. Die Injektion in die Mitte des Lobulus 4. des Lobi ant., wo In $\mathrm{g} v \mathrm{a} \mathrm{r}^{\mathrm{5}}$ ) die Koordinationszentren für den Nacken und die vorderen Extremitäten lokalisiert sah, führte in den meisten Fällen neben der eben genannten Haltungsanomalie der vorderen Extremitäten noch ein Herabhängen des Kopfes herbei, sodass sich dieser auf die vorderen Extremitäten legte. Beim Aufheben des Kopfes an den Ohrmuscheln leistete der Hals keinen nennenswerten Widerstand. Half man dem Tier durch Unterstützung der Brust auf, sodass sich die vorderen Extremitäten ungefähr in normaler Haltung befanden, so hing der Kopf zwischen diesen Extremitäten schlaff herab. Dieses Herabhängen des Kopfes konnte in seltenen Fällen der Verschiebung der vorderen Extremitäten eine kurze Zeit vorangehen. Nach der Injektion in die Mitte des Lobulus C. des Lobi post., wo nach $\mathrm{Narumi} \mathrm{i}^{2}$ das Koordinationszentrum für die hinteren Extremitäten zu suchen ist, erfolgte eine Haltungsanomalie dieser Extremitäten, die in einem Strecken der Unterschenkeln und der Füsse bestand, sodass sich diese ausserordentlich stark nach vorn verschoben. Während dieser Erscheinungen liess sich weder in der Respiration noch bei den Herzschlägen eine wesentliche Veränderung erkennen, woraus hervorgeht, dass sie nicht durch resorptive, sondern durch örtliche Wirkung des Giftes auf die betreffenden Gehirnteile entstehen.

Diese Versuche deuten darauf hin, dass die bei der intravenösen Darreichung des Digitoxins erfolgenden Haltungsanomalien des Kopfes und der Extremitäten von der Wirkung des Giftes auf die genannten Koordinationszentren im Kleinhirn herrühren.

Aus obigen Versuchen ergibt sich, dass das Digitoxin, vielleicht auch die anderen Digitaliskörper bei Kaninchen besonders auch auf das Kleinhirn einwirkt und zuerst das Koordinationszentrum für den Nacken im Lobulus 4. des Lobi ant., dann die Koordinationszentren für die vorderen Extremitäten in diesem und im Lobuli simp., und zuletzt das Koordinationszentrum für die hinteren Extremitäten im Lobulus C. des Lobi post. ausser Tätigkeit setzt, sodass bei intravenöser Vergiftung zuerst ein Herabhängen des Kopfes, dann eine Vorwärtsverschiebung der vorderen Extremitäten und zuletzt eine solche der hinteren erfolgt.

5) Ingva r, Folia neurobiolog., 1920, 11, 205. 